\title{
BMJ Open Preferences for portable ultrasound devices: a discrete choice experiment among abdominal aortic aneurysm surveillance patients and general ultrasound patients in England
}

\author{
Caron Parsons, ${ }^{1,2}$ Kamran Ahmad Khan, ${ }^{1}$ Joshua Pink, ${ }^{3}$ Alice Verran, ${ }^{1}$ \\ Frances Griffiths, ${ }^{1}$ Charles E Hutchinson, ${ }^{1,2}$ Stavros Petrou ${ }^{1}$
}

To cite: Parsons C, Khan KA Pink J, et al. Preferences for portable ultrasound devices: a discrete choice experiment among abdominal aortic aneurysm surveillance patients and general ultrasound patients in England. BMJ Open 2018;8:e025428. doi:10.1136/ bmjopen-2018-025428

- Prepublication history and additional material for this paper are available online. To view these files, please visit the journal online (http://dx.doi org/10.1136/bmjopen-2018025428).

Received 14 July 2018 Revised 24 October 2018 Accepted 16 November 2018

Check for updates

(c) Author(s) (or their employer(s)) 2018. Re-use permitted under CC BY-NC. No commercial re-use. See rights and permissions. Published by BMJ.

${ }^{1}$ Warwick Medical School, University of Warwick, Coventry, UK

${ }^{2}$ Department of Radiology, University Hospitals Coventry and Warwickshire, Coventry, UK ${ }^{3}$ National Institute for Health and Care Excellence, Manchester, UK

Correspondence to

Dr Caron Parsons;

c.s.parsons@warwick.ac.uk

\section{ABSTRACT}

Objective To undertake an assessment of preferences as to how, where and by whom ultrasounds (US) should be performed in: (1) patients undergoing surveillance of abdominal aortic aneurysm (AAA) size (AAA group); and (2) patients being scanned for general abdominal conditions (general group).

Design A discrete choice experiment (DCE) questionnaire was administered to patients attending US appointments. Analysis of questionnaire responses used conditional logit models and included validity checks.

Setting West Midlands, England.

Participants 524 patients (223 in the AAA group and 301 in the general group) were recruited from the US outpatient department at University Hospital Coventry and Warwickshire.

Outcome measures Coefficients for attributes in relation to their reference levels.

Results The AAA group preferred to have their US performed in hospital while the general group had a preference for portable US at general practice surgeries. All patients had a strong preference for scanning by specialists, devices with a lower risk of underdiagnosis and receiving their results at the appointment where the scan takes place. The general group had a strong preference for the person performing the scan to know their medical history.

Conclusions Patients being scanned for general abdominal conditions prefer to be scanned in a general practice by practitioners who know their medical history. Patients undergoing surveillance of AAA size prefer to be scanned in a hospital setting. Both groups would prefer to be informed of the scan results as soon as possible. Further research is required to explore the clinical scenarios in which targeted scanning by community practitioners would be of benefit to patients.

\section{INTRODUCTION}

There has been a steep rise in the volume of requests for imaging tests across the developed world. In the UK, there was a $4.2 \%$ annual increase in the number of ultrasound

\section{Strengths and limitations of this study}

- The study has a sample size meeting the requirements of statistical efficiency and response efficiency which enabled validity checks and allowed for subgroup comparisons.

- The multistage approach to the selection of attributes and levels was thorough including an expert group scoping exercise, semistructured interviews with participants followed by a ranking exercise carried out with a separate group of participants.

- Participants were recruited within an outpatient setting of a single centre and are therefore not necessarily representative of the general population.

- The average age of the general ultrasound population was 65.09 years which was deliberately matched to the abdominal aortic aneurysm population, potentially limiting the applicability to a younger general population.

- The study was not designed to examine patient attitudes towards false-positive results, but clearly this should be evaluated in future research.

(US) requests between 2012 and 2017, resulting in 9.28 million requests for US examinations in 2016-2017. ${ }^{2}$ Given that this increase in requests has not been mirrored by a rise in the number of radiologists or ultrasonographers in the UK, ${ }^{34}$ alternative solutions for managing requests are required. The rise in requests has been accompanied by growth in the portable US market with a wide range of devices now available. There are financial and logistical arguments for performing diagnostic tests in the community which may be facilitated by the use of portable devices.

There are several portable US devices available on the market which are capable of testing for abdominal aortic aneurysms (AAA), one of which is the 'VScan' which is a small hand-held US device developed and 
produced by General Electric (GE Healthcare). Several studies have demonstrated the diagnostic accuracy of US performed by general practitioners and other non-radiological practitioners in the assessment of left ventricular function (sensitivity $92 \%$, specificity $94 \%$ ), ${ }^{5}$ postoperative evaluation of pleural and pericardial effusions (sensitivity $91 \%-98 \%$, specificity $56 \%-70 \%)^{6}$ and in AAA identification (sensitivity $100 \%$, specificity $100 \%$ ). ${ }^{7}$ However, diagnostic accuracy represents only one strand of evidence that should inform healthcare decision-making and should be used in conjunction with evidence for cost-effectiveness and patient preferences. ${ }^{8}$

Discrete choice experiments (DCEs) are increasingly used by economists and psychologists to inform healthcare policy decisions. They measure the relative importance that respondents place on different characteristics of a service or product, and the extent to which they are prepared to forfeit one characteristic to have more of another. DCEs are based on the premise that a service can be described in terms of attributes, each of which can be set at different levels. In a DCE, participants have to choose between scenarios with differing levels of these attributes. Regression techniques yield coefficients that describe the relative preference (utility) for changes in each attribute. Coefficient ratios provide information on responders' willingness to trade between attributes. ${ }^{9} 10$ The increasing use of DCEs in healthcare arises from the recognition that healthcare interventions are valued for more than just health outcomes. ${ }^{10}{ }^{11}$ DCEs have been successfully used to involve the public and patients in establishing preferences for health care. ${ }^{12-15}$

Patient experience and preference has become increasingly important in decision-making in healthcare policy. ${ }^{16}$ Very little is currently known about patient preferences on by whom, how and where imaging tests should be performed. Surveys and questionnaires have been used in the past to evaluate patient preferences as to how results of imaging tests are conveyed, and how much the radiologist should be involved in this process; however, DCE methodologies that permit an estimation of strength of preference were not applied. ${ }^{18-20}$ Attitudes to findings of extracolonic malignancy in CT colonography ${ }^{21}$ have been evaluated using DCE techniques, but there has been no evaluation of patient preferences for general abdominal US or AAA surveillance.

The aim of this study was to evaluate two groups of patients (undergoing AAA surveillance or general abdominal US) and their preferences for how, by whom and where their US should be performed, using the DCE technique.

\section{METHODS}

This study was conducted using an exploratory sequential mixed-methods design, adopting recommended stages for undertaking a DCE. ${ }^{22}$ In selecting methods to design the questionnaire and conduct the DCE, reference was made to the International Society for Pharmacoeconomics and
Outcomes Research good practice checklist for conjoint analysis. ${ }^{23}$

\section{Selection of attributes and levels}

Qualitative work should be undertaken to inform the development of attributes and levels for DCEs. This ensures that attributes that are important to patients or potential recipients are included and provides a way of checking that respondents are likely to understand the attributes they are presented with in the way that researchers expect. ${ }^{9} 1024$ A multistage approach was used to develop the attributes and associated levels.

\section{Expert group}

A scoping exercise was conducted, and a list of topics was produced. An expert group $(n=4)$ was convened including a range of professionals with expert knowledge of the service and technology context. The group discussed the list of topics and was asked to suggest an initial set of attributes and levels to be used in semistructured interviews.

\section{Semistructured interviews}

Semistructured, qualitative interviews were conducted with patients attending US appointments to identify which themes and factors they consider important in how their scan is performed. Convenience sampling was used and two groups were interviewed: one attending for general abdominal US $(n=14)$, the other for AAA monitoring $(n=9)$. Prior to interview, the AAA group had undergone two scans: one carried out by a vascular nurse specialist using standard US equipment, the other performed by a general practitioner (GP) using the VScan portable US machine. Inclusion criteria were age over 50 years and attendance for either AAA monitoring or abdominal US. Patients were excluded if they did not speak English, and there was no available interpreter. Interviews lasted 15 min and explored participants' experiences and views regarding factors important to them such as the location of scan and who performs it. Participants were then provided with the initial attribute list, asked whether these were relevant and to identify any additional attributes important to them. The interviews were audio-recorded and later transcribed; the transcriptions were then checked for accuracy. Thematic analysis was carried out on the interview data to produce a more comprehensive list of potential attributes and levels.

\section{Ranking exercise to select attributes}

Convenience sampling was used to identify participants for a separate ranking exercise. Interviews were held with patients attending US appointments $(\mathrm{n}=17)$. Participants were initially provided with the updated list of attributes and asked to think of any that were also important to them. This was followed by the completion of a ranking exercise in which participants were asked to select the five most important attributes from a list of 14 and then rank these attributes in order of importance. Participants were given the opportunity to clarify anything they did 
Box $1 \mathrm{~A}$ Discrete choice experiment attributes and levels

\section{Knowledge of your health}

a. The person performing your scan has knowledge of your health/ other health problems.

b. The person performing your scan does not have prior knowledge of your health/other health problems.

\section{Person scanning has specialist training in scanning for aortic aneurysm}

a. The practitioner performing your scan has detailed knowledge of the National Health Service (NHS) Abdominal Aortic Aneurysm Screening Programme.

b. The practitioner performing your scan does not have detailed knowledge of the NHS Abdominal Aortic Aneurysm Screening Programme.

\section{Continuity of care}

a. You see the same practitioner each time you go for a scan.

b. You see different practitioners each time you go for a scan.

Risk of underdiagnosis (chance that the scan will give an incorrect negative result, meaning any potential problem would not be picked up until your next scan)

a. $2 \%(1$ in 50$)$.

b. $5 \%(1$ in 20$)$.

\section{Time taken to receive your results}

a. You get your results at the appointment where your scan takes place.

b. Your get your results later via a report from the NHS Abdominal Aortic Aneurysm Screening programme.

not understand. The data from this ranking exercise were analysed and subsequently used to inform the final design of the DCE.

\section{Questionnaire design}

A labelled choice format for the DCE was selected as this may be more suitable to real-life choices such as uptake of cancer screening. ${ }^{25}$ The final design comprised six attributes each with two levels (box 1A) and the referent level for each attribute is described in box 1B. A generic pairwise choice with an 'opt-out' choice was selected for the questionnaire design. Each scenario described a choice between two possible screening alternatives. In each choice set, participants were asked to select their preferred choice for screening from three options, one of which was a 'neither' option (see table 1 for an example choice question). There was no cost attribute included in the study as the National Health Service (NHS) provides US scanning free at the point of use.

The DCE design was generated using SAS (V.8.0) statistical software. The most efficient design based on the properties of orthogonality and level balance consisted of 12 separate choice sets, each comprising two alternative profiles.

Due to the small number of choice sets, they were all incorporated into a single questionnaire to be administered to all participants. One questionnaire was designed with wording altered for each group: one for patients undergoing surveillance scanning of their AAAs, and one
Box 1B Reference cases for the abdominal aortic aneurysm (AAA) population and the general abdominal ultrasound (US) population

Reference case for the AAA population:

- Select option A.

- Traditional hospital-based US scanner, operated by a full-time ultrasonographer.

- The person performing your scan does not have prior knowledge of your health/other health problems.

- The practitioner performing your scan does not have detailed knowledge of the National Health Service Abdominal Aortic Aneurysm Screening Programme.

- You see different practitioners each time you go for a scan.

- Risk of underdiagnosis: $2 \%$ (1 in 50).

- You get your results at the appointment where your scan takes place.

\section{Reference case for the general US population:}

- Select option A.

- Traditional hospital-based US scanner, operated by a full-time ultrasonographer.

- The person performing your scan does not have prior knowledge of your health/other health problems.

- The practitioner performing your scan is not a specialist US scanner (eg, GP/practice nurse).

- You see different practitioners each time you go for a scan.

- Risk of underdiagnosis: $2 \%$ (1 in 50 ).

- You get your results at the appointment where your scan takes place.

for patients who had been referred for general outpatient abdominal US scans. Core questions additionally collected descriptive data on each participant.

Each version of the questionnaire included one consistency test. The consistency test was identical to, but not consecutive with, a selected choice set from the set of questions. Responders were deemed to have answered consistently if they selected the same option in the original choice set and the consistency test.

\section{Participants and recruitment procedure}

Participants were recruited from the US outpatient department at University Hospital Coventry and Warwickshire between February 2015 and May 2016. A research nurse identified whether a patient was attending for general abdominal US or AAA screening follow-up scan and gained consent from those willing to participate in the study. Only patients above the age of 60 years were approached for recruitment into the general group. For the purposes of eliciting the preferences of patients attending for general abdominal US or AAA screening follow-up scan, a prespecified sample size of at least 200 patients in each group was required to meet the requirements of statistical efficiency and response efficiency. ${ }^{26}$

Questionnaires were provided to patients, and they were given the option of completing it and returning it at the time of the US appointment or returning the questionnaire by post. Participants were offered a contribution 
Table 1 Example of a choice question

Q1: Which of the two alternative screening programmes shown below, or neither, would you choose to attend?

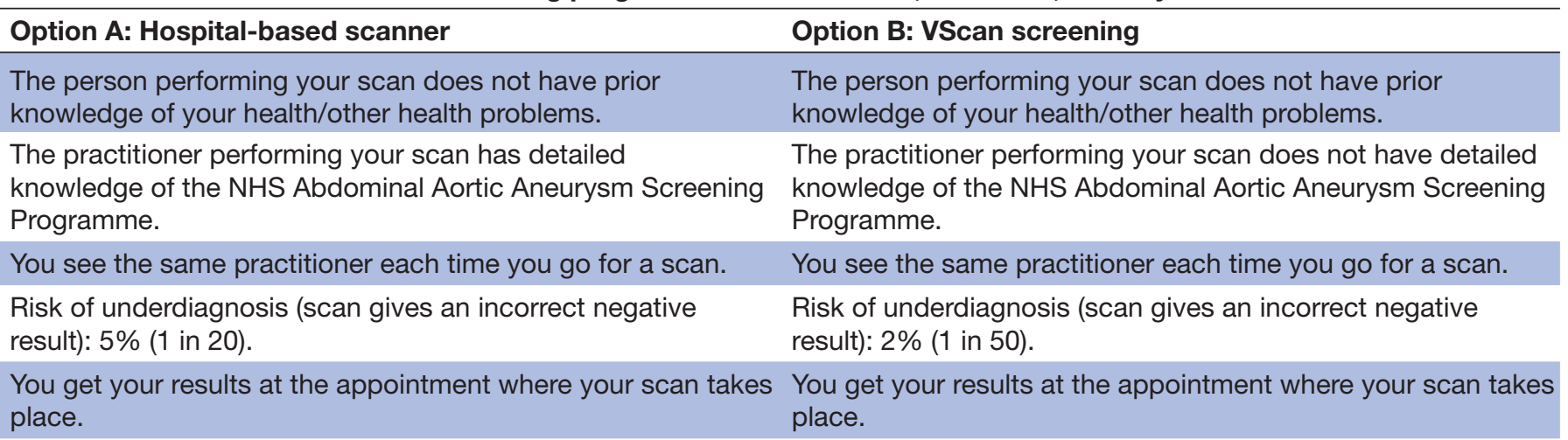

Please tick the box below which corresponds to which of the two screening alternatives, or neither, you would choose to attend. Please select only one option.

\begin{tabular}{lc} 
Option A & $\square$ \\
Option B & $\square$ \\
Neither & $\square$ \\
\hline
\end{tabular}

NHS, National Health Service.

to parking/travelling costs to the value of a $£ 5$ shopping voucher for questionnaire completion.

\section{Patient and public involvement}

Patients were involved in design aspects of two parts of the study as described in detail above. The first group $(n=31)$ were interviewed by a researcher and were asked what attributes of attending a US were important to them, based on an initial list formulated by the expert group. The second separate group $(n=17)$ were asked to rank the attributes in order of importance. This information was then incorporated into the design of the final DCE questionnaire. The outcomes of the study will be made available to participants.

\section{Statistical analyses}

Questionnaire responses were entered independently into MS Excel (V.2013) by two researchers, thus creating two sets of data. Discrepancies were resolved by referral to source questionnaires. Descriptive patient characteristics were analysed using frequencies. DCE data were transferred to R (V.3.4.1) and analysed using conditional logit regression analysis, ${ }^{9}$ clustered on participant ID. Categorical data were expressed as dummy variables. The two sets of data input were compared for differences. Internal validity was evaluated by comparing the main results with those of respondents that answered the consistency check question accurately. Additional sensitivity analyses were conducted to see if the results of the DCE varied by age or gender. Marginal rates of substitution were calculated by using risk of underdiagnosis as the continuous variable.

Regression coefficients were calculated for all attributes in the regression model. A negative coefficient represents a preference for the referent level whereas a positive coefficient represents a preference for the alternative presented in the results table. The magnitude of the regression coefficients represented the degree of preference for each of the attributes: the greater the coefficient, the more that attribute was preferred. Ratio of coefficients was used to demonstrate how respondents traded between attributes. The ratio of the risk coefficient to each of the non-risk coefficients was used to calculate willingness to trade for changes in the non-risk attributes.

Sensitivity analyses were conducted to see if the results of the DCE varied by age or gender.

Age was entered as a categorical variable split based on median age ( 1 is above median $(\mathrm{AAA}=(75-94)$, general $=(64-94)), 0$ is less than or equal to median $(\mathrm{AAA}=(50-74)$, general $=(50-63))$, while gender was entered as a categorical variable $(0=$ male, $1=$ female $)$.

\section{RESULTS}

\section{Characteristics of population}

The sample for the DCE comprised 524 respondents (223 AAA patients and 301 general abdominal US patients). The general group included patients who had been referred by their general practitioner or hospital consultant for any type of abdominal health concern. The general characteristics of both populations are presented in table 2. There was no significant difference between the ages of the two patient populations. There was a significantly higher proportion of men in the AAA surveillance group.

\section{Main DCE results}

AAA group

The coefficients for the full dataset are presented in table 3, along with their SEs and significance. Patients 
Table 2 Characteristics of the two patient populations

\begin{tabular}{llll}
\hline & AAA patients & $\begin{array}{l}\text { General } \\
\text { ultrasound }\end{array}$ & P value* $^{*}$ \\
\hline $\mathrm{N}$ & 223 & 301 & \\
$\begin{array}{l}\text { Age (years), } \\
\text { mean (SD), }\end{array}$ & $71.04(10.18)$, & $65.09(10.87)$, & $\mathrm{P}=0.8500$ \\
$\begin{array}{l}\text { median } \\
\begin{array}{l}\text { Gender (women), } \\
\mathrm{n}(\%)\end{array}\end{array}$ & 63 & \\
\hline
\end{tabular}

*T test for continuous variable and $\chi^{2}$ test for proportions.

AAA, abdominal aortic aneurysm.

expressed the strongest preference for a device with a lower risk of underdiagnosis $(-0.553 ; \mathrm{p}<0.001)$. The residual coefficient for the practitioner performing the scan having detailed knowledge of the AAA programme was significant and positive which signifies that patients have a strong preference for practitioners that are specialists in scanning $(0.430 ; \mathrm{p}<0.001)$. Location of scan was the attribute showing the next strongest preference with AAA patients having a preference for hospital scanning $(-0.216 ; \mathrm{p}<0.001)$. The coefficient for speed of results was negative and significant, indicating that AAA patients prefer to receive their results at the appointment where the scan takes place $(-0.175 ; \mathrm{p}<0.01)$. These results are consistent with the expectation that AAA patients will prefer a scan at the hospital by a specialist using a more accurate device with immediate feedback of results.

\section{General group}

The coefficients for the full dataset are presented in table 4, along with their SEs and estimates of statistical significance. Patients expressed the strongest preference for a device with a lower risk of underdiagnosis $(-0.455 ; \mathrm{p}<0.001)$. The residual coefficient for the practitioner performing scan having detailed knowledge of the AAA programme was significant and positive which signifies that patients have a strong preference for practitioners that are specialists in scanning
$(0.449 ; \mathrm{p}<0.001)$. Location of scan was the attribute showing the next strongest preference with general US patients preferring scanning at a GP surgery $(0.309 ; \mathrm{p}<0.001)$, in contrast to the AAA patients who preferred to have their scan in the hospital. The coefficient for speed of results was negative and significant, indicating that general US patients prefer to receive their results at the appointment where the scan takes place $(-0.138 ; p<0.01)$. General US patients also have a preference to be seen by healthcare practitioners with more knowledge of their health $(0.130 ; \mathrm{p}<0.01)$.

Internal validity checks showed that, in comparison with the full dataset, all checks yielded very similar results with no change in the order of the strength of preference for a level within each attribute. Results of the internal validity checks are included in the online supplementary tables S1 and S2.

\section{Sensitivity analyses \\ AAA group}

There were no differences in preferences for seeing the same person, being cared for by healthcare practitioners with more knowledge of their health, being cared for by specialists in scanning and receiving results at the appointment where the scan takes place among the AAA respondents, by age group or sex. When looking at the result for preferences for devices with a lower risk of underdiagnosis in the AAA population, there is a suggestion that younger respondents cared more about the risk of underdiagnosis and women cared slightly less than men about the risk of underdiagnosis (online supplementary tables S3(A-E) and S4 (A-E)).

\section{General group}

There were no differences by age group or sex in the preferences of patients scanned for general abdominal conditions for seeing the same person, being cared for by healthcare practitioners with more knowledge of their health, being cared for by specialists in scanning and receiving their results at the appointment where the scan takes place. There was also no difference in the

\begin{tabular}{|c|c|c|c|}
\hline Characteristic & Coefficient & SE (coefficient) & Significance \\
\hline Selecting option B (second choice set). & 0.129 & 0.061 & * \\
\hline VScan device at a local GP surgery. & -0.216 & 0.053 & *** \\
\hline $\begin{array}{l}\text { The person performing your scan has knowledge of your health/other health } \\
\text { problems. }\end{array}$ & 0.084 & 0.055 & \\
\hline $\begin{array}{l}\text { The practitioner performing your scan has detailed knowledge of the NHS } \\
\text { Abdominal Aortic Aneurysm Screening Programme. }\end{array}$ & 0.430 & 0.055 & *** \\
\hline You see the same practitioner each time you go for a scan. & 0.040 & 0.057 & \\
\hline Risk of underdiagnosis: $5 \%$ (1 in 20). & -0.553 & 0.055 & $* \star \star$ \\
\hline $\begin{array}{l}\text { You get your results later via a report from the NHS Abdominal Aortic } \\
\text { Aneurysm Screening programme. }\end{array}$ & -0.175 & 0.054 & ** \\
\hline
\end{tabular}

${ }^{*} \mathrm{P}<0.05,{ }^{* *} \mathrm{P}<0.01,{ }^{* * *} \mathrm{P}<0.001$.

AAA, abdominal aortic aneurysm; NHS, National Health Service. 
Table 4 General ultrasound population main results

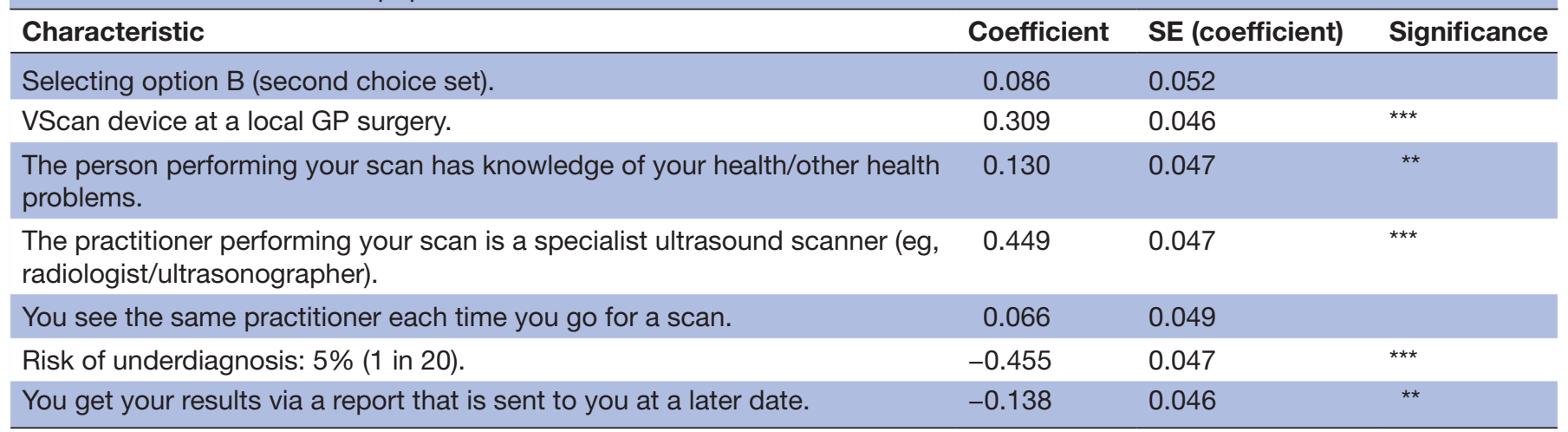

${ }^{*} \mathrm{P}<0.05,{ }^{* \star} \mathrm{P}<0.01,{ }^{* \star *} \mathrm{P}<0.001$.

preference for devices with a lower risk of underdiagnosis by age group. When looking at the preferences for devices with a lower risk of underdiagnosis by gender, we found that women cared slightly more than men about the risk of underdiagnosis (online supplementary tables S5(A-E) and S6(A-E)).

\section{Marginal rate of substitution}

An individual from the AAA population would, on average, need to have the risk of underdiagnosis fall by $1.17 \%$ to be prepared to have a scan at a general practice surgery rather than in hospital (online supplementary tables S7-8). In contrast, an individual from the general US population would, on average, require the risk of underdiagnosis to increase by $2.04 \%$ to be prepared to have a scan at hospital.

An individual from the general US population would require a greater increase in risk of underdiagnosis to be prepared to have the scan performed by someone without knowledge of their health problems or to be scanned by a different practitioner each time they go for a scan.

An individual from the AAA population would require a larger reduction in risk of underdiagnosis, before they would change preference and wait for results via a later report (online supplementary tables S7-8).

\section{DISCUSSION}

Patients have a strong preference for specialists in scanning, devices with a lower risk of underdiagnosis and receiving their results immediately after the scan takes place. AAA patients are not as concerned about who performs their scan, whereas general US patients have a strong preference for the practitioner performing the scan to understand their health problems.

In both groups studied, the residual coefficient for the VScan device versus hospital scanners is significant, but the direction of effect is different. AAA patients prefer hospital scanning, while abdominal US patients prefer the VScan device at general practice surgeries. The preference for a hospital scan by the AAA patients is unsurprising, given that they are under the care of a specialist team and require information on the size of their aneurysm and how close this measurement takes them to needing potential surgery. General US patients do not usually have the same levels of anxiety as AAA patient ${ }^{27}$ and have usually been referred by their GP. It is logical that this population would want their GP to amalgamate knowledge of their general health with a diagnostic test as quickly and easily as possible.

This DCE demonstrates the importance of test accuracy for all patients. The expertise of personnel performing the scan and receiving the scan results promptly is important to both groups of patients. AAA surveillance patients prefer to have their scans performed in hospital, whereas general abdominal US patients would prefer to have a scan at a general practice. The two groups also differed in their preferences for the type of professional that performed the scan, with respect to knowledge of the patient's medical history. Given that AAA patients will have seen a vascular surgical team, it is unsurprising that they are less concerned with how well acquainted the ultrasonographer is with their medical history. Conversely, general abdominal US patients value the practitioner having knowledge of their medical history.

This study is, to our knowledge, the first of its kind to offer quantitative evidence of patient preferences for scanning in AAA surveillance and in general abdominal presentations. Strengths of the study include a sample size meeting the requirements of statistical efficiency and response efficiency ${ }^{26}$ which enabled validity checks and allowed for subgroup comparisons. The multistage approach to the selection of attributes and levels was thorough: semistructured interviews allowed exploration of ideas important to participants and the interviews appeared to give data saturation.

This DCE study has several limitations. These mostly relate to the populations examined; all patients were recruited within the outpatient setting of a single centre, and therefore not necessarily representative of the general population. The average age of the general US population was 65.09 years which was deliberately matched to the AAA population, potentially limiting the applicability 
to a younger general population. Clearly, patients, who did not attend their appointment during the recruitment period and those who declined to participate in the study, may differ from the two groups who participated in the study leading to the potential for self-selection bias. The study was not designed to examine patient attitudes towards false-positive results, but clearly this should be evaluated in future research.

It is difficult to make a direct comparison with other published DCEs in the radiological arena, as there is little comparative published literature. Plumb et $a l^{21}$ demonstrated an extremely high tolerance for the diagnosis of false-positive extracolonic malignancies in CT colonography, leading to unnecessary additional radiological or invasive tests despite face-to-face interviews providing background information via interactive laptop presentation. Our study evaluated the importance of false-negative results to patients, but was not designed to explore patients' understanding of false-positive results. Screening studies have also demonstrated the relatively poor understanding that patients can have of test accuracy. ${ }^{28} 29$

Although previous studies have examined patient preferences to how, by whom and when results are conveyed to patients, results have been mixed and the methodologies used have not been based on robust qualitative or economic methods. Schreiber et al, ${ }^{18}$ found that patients prefer to have their imaging results conveyed directly by the radiologist, whereas Mangano $e t a l^{19}$ found that patients preferred to have detailed examination results from the requesting physician. Some of these conflicting results may relate to the fact that the results may be delivered more quickly if delivered by a radiologist, but may not lead to a detailed discussion of the impact of the results on the patient.

The diagnostic capacity of portable US devices has not been fully evaluated in general abdominal conditions. Thus far, portable US has undergone thorough evaluation in cardiac and thoracic pathologies. However, its potential for relatively simple or binary clinical scenarios such as presence of gallstone disease, bladder distension and hydronephrosis has not been evaluated. Portable US performed by general practitioners in these scenarios could potentially benefit patients, both by saving time and cost, but also by satisfying patients' preference for a scan in the community by their doctor, who has knowledge of their medical history. Clearly, the value of this scenario needs to be set in the context of the potential limitations such as the potential for false-positives and potentially suboptimal operator accuracy which should inform the discussion between the general practitioner and patient.

The difficulty of travel to and from Trusts in the UK as well as the challenges and costs of parking are well documented and lead to non-attendance and its concomitant economic implications. ${ }^{30}$ There are therefore potential cost-savings to the patient and the healthcare system (via reduced travel, parking costs and time off work) by substituting a hospital-based service with a community-delivered US service at the time of patient presentation. Other populations that may benefit from portable US may reside in geographically remote or deprived areas. Studies have demonstrated decreased uptake of AAA screening in socially deprived and urban areas, ${ }^{31}$ and therefore general practitioners using portable US could target certain populations or areas at a local level. ${ }^{32}$

In conclusion, this large study has demonstrated the importance of test accuracy to two different patient groups. It is one of the first studies to use DCE methodology in the radiological arena and results particularly emphasise that general abdominal US patients prefer scanning in the community by practitioners who have an understanding of their medical history. The patient preferences elicited by this study should be considered in conjunction with other sources of evidence including diagnostic accuracy and cost-effectiveness when considering the adoption of portable US in routine clinical practice.

Acknowledgements The authors wish to thank all the patients who took the time to participate in the interviews and complete a detailed questionnaire describing their preferences.

Contributors JP undertook the DCE design and analysis. KAK carried out the DCE analyses. SP supervised the DCE design and analysis. FG supervised the qualitative research. AV carried out the qualitative research. CEH and CP provided clinical input into the development of the DCE materials. CP supervised recruitment into the study. KAK, JP, SP, CEH, CP, FG and AV wrote the first draft of the article. CP, JP and SP wrote the second draft of the article. All authors contributed to and agreed the final version of the manuscript. All authors had full access to all of the data (including statistical reports and tables) in the study and can take responsibility for the integrity of the data and the accuracy of the data analysis.

Funding The initial study was funded by NHS England Regional Innovation Fund (28560) and GE Healthcare. The VScan devices were on short-term loan from GE Healthcare.

Competing interests GE Healthcare was not involved in the planning of the discrete choice experiments.

Patient consent for publication Not required.

Ethics approval Ethical approval was granted by NRES committee West Midlands, Edgbaston (13/WM/0022).

Provenance and peer review Not commissioned; externally peer reviewed.

Data sharing statement Individual-level data from the study are available from the corresponding author on reasonable request.

Open access This is an open access article distributed in accordance with the Creative Commons Attribution Non Commercial (CC BY-NC 4.0) license, which permits others to distribute, remix, adapt, build upon this work non-commercially, and license their derivative works on different terms, provided the original work is properly cited, appropriate credit is given, any changes made indicated, and the use is non-commercial. See: http://creativecommons.org/licenses/by-nc/4.0/.

\section{REFERENCES}

1. England N. Diagnostic Imaging Dataset Statistical Release, 2013.

2. England N. Diagnostic Imaging Dataset Statistical Release, 2018.

3. Clinical radiology UK workforce census 2016 report. London: The Royal College of Radiologists, 2017.

4. The Society and College of Radiographers. Sonographer Workforce Survey Analysis, 2014.

5. Mjølstad OC, Andersen GN, Dalen $\mathrm{H}$, et al. Feasibility and reliability of point-of-care pocket-size echocardiography performed by medical residents. Eur Heart J Cardiovasc Imaging 2013;14:1195-202.

6. Graven T, Wahba A, Hammer AM, et al. Focused ultrasound of the pleural cavities and the pericardium by nurses after cardiac surgery. Scand Cardiovasc J 2015;49:56-63.

7. Kuhn M, Bonnin RL, Davey MJ, et al. Emergency department ultrasound scanning for abdominal aortic aneurysm: accessible, accurate, and advantageous. Ann Emerg Med 2000;36:219-23.

8. Excellence NIfHaC. Diagnostic Assessment Programme Manual. Lonson, UK: NICE, 2011. 
9. Ryan M, Gerard K, Amaya-Amaya M, eds. Using discrete choice experiments to value health and health care: Springer Academic Publishers, 2008.

10. Lancsar E, Louviere J. Conducting discrete choice experiments to inform healthcare decision making: a user's guide. Pharmacoeconomics 2008;26:661-77.

11. Ryan M. Using conjoint analysis to take account of patient preferences and go beyond health outcomes: an application to in vitro fertilisation. Soc Sci Med 1999;48:535-46.

12. Hall J, Kenny P, King M, et al. Using stated preference discrete choice modelling to evaluate the introduction of varicella vaccination. Health Econ 2002;11:457-65.

13. Porteous T, Ryan M, Bond CM, et al. Preferences for self-care or professional advice for minor illness: a discrete choice experiment. Br J Gen Pract 2006;56:911-7.

14. Sculpher M, Bryan S, Fry P, et al. Patients' preferences for the management of non-metastatic prostate cancer: discrete choice experiment. BMJ 2004;328:382.

15. Watson V, Ryan M, Watson E. Valuing experience factors in the provision of Chlamydia screening: an application to women attending the family planning clinic. Value Health 2009;12:621-3.

16. Say RE, Thomson R. The importance of patient preferences in treatment decisions-challenges for doctors. BMJ 2003;327:542-5.

17. Siminoff LA. Incorporating patient and family preferences into evidence-based medicine. BMC Med Inform Decis Mak 2013;13:S6.

18. Schreiber MH, Leonard M, Rieniets CY. Disclosure of imaging findings to patients directly by radiologists: survey of patients' preferences. AJR Am J Roentgenol 1995;165:467-9.

19. Mangano MD, Rahman A, Choy G, et al. Radiologists' role in the communication of imaging examination results to patients: perceptions and preferences of patients. AJR Am J Roentgenol 2014;203:1034-9.

20. Pahade JK, Trout AT, Zhang B, et al. What patients want to know about imaging examinations: a multiinstitutional u.s. survey in adult and pediatric teaching hospitals on patient preferences for receiving information before radiologic examinations. Radiology 2018;287:554-62.

21. Plumb AA, Boone D, Fitzke $\mathrm{H}$, et al. Detection of extracolonic pathologic findings with CT colonography: a discrete choice experiment of perceived benefits versus harms. Radiology 2014;273:144-52.

22. Ryan M, Gerard K, Amaya-Amaya M. Using discrete choice experiments to value health and health care. Springer: Dordrecht, 2008.

23. Bridges JF, Hauber AB, Marshall D, et al. Conjoint analysis applications in health-a checklist: a report of the ISPOR Good Research Practices for Conjoint Analysis Task Force. Value Health 2011;14:403-13.

24. Coast J, Al-Janabi H, Sutton EJ, et al. Using qualitative methods for attribute development for discrete choice experiments: issues and recommendations. Health Econ 2012;21:730-41.

25. de Bekker-Grob EW, Hol L, Donkers B, et al. Labeled versus unlabeled discrete choice experiments in health economics: an application to colorectal cancer screening. Value Health 2010;13:315-23.

26. Reed Johnson F, Lancsar E, Marshall D, et al. Constructing experimental designs for discrete-choice experiments: report of the ISPOR Conjoint Analysis Experimental Design Good Research Practices Task Force. Value Health 2013;16:3-13.

27. Hansson A, Brodersen J, Reventlow S, et al. Opening Pandora's box: The experiences of having an asymptomatic aortic aneurysm under surveillance. Health Risk Soc 2012;14:341-59.

28. Domenighetti G, D'Avanzo B, Egger M, et al. Women's perception of the benefits of mammography screening: population-based survey in four countries. Int J Epidemiol 2003;32:816-21.

29. Barratt A, Cockburn J, Furnival C, et al. Perceived sensitivity of mammographic screening: women's views on test accuracy and financial compensation for missed cancers. J Epidemiol Community Health 1999;53:716-20.

30. GOV.UK. HTM 07-03 NHS car-parking management: environment and sustainability, 2015.

31. Crilly MA, Mundie A, Bachoo P, et al. Influence of rurality, deprivation and distance from clinic on uptake in men invited for abdominal aortic aneurysm screening. Br J Surg 2015;102:916-23.

32. Jacomelli J, Summers L, Stevenson A, et al. Editor's choice inequalities in abdominal aortic aneurysm screening in england: effects of social deprivation and ethnicity. Eur J Vasc Endovasc Surg 2017;53:837-43. 